\title{
Hunting for dark matter subhalos among the Fermi-LAT sources with VERITAS
}

\section{Daniel Nieto* for the VERITAS Collaboration ${ }^{\dagger}$}

Columbia University - Nevis Laboratories

E-mail: nieto@nevis.columbia.edu

The distribution of dark matter in the Galaxy, according to state-of-the-art simulations, shows not only a smooth halo component but also a rich substructure where a hierarchy of dark matter subhalos of different masses is found. We present a search for potential dark matter subhalos in our Galaxy exploiting the high (HE, $100 \mathrm{MeV}-100 \mathrm{GeV}$ ) and very-high-energy (VHE, >100 $\mathrm{GeV}$ ) $\gamma$-ray bands. We assume a scenario where the dark matter is composed of weakly interacting massive particles of mass over $100 \mathrm{GeV}$, and is capable of self-annihilation into standard model products. Under such a hypothesis, most of the photons created by the annihilation of dark matter particles are predicted to lay in the HE $\gamma$-ray band, where the Fermi-Large Area Telescope is the most sensitive instrument to date. However, the distinctive spectral cut-off located at the dark matter particle mass is expected in the VHE $\gamma$-ray band, thus making imaging atmospheric Cherenkov telescopes like VERITAS the best suited instruments for follow-up observations and the characterization of a potential dark matter signature. We report on the ongoing VERITAS program to hunt for these dark matter subhalos, particularly focusing on two promising dark matter subhalo candidates selected among the Fermi-LAT Second Source Catalog unassociated high-energy $\gamma$-ray sources.

The 34th International Cosmic Ray Conference,

30 July- 6 August, 2015

The Hague, The Netherlands

${ }^{*}$ Speaker. $\quad{ }^{\dagger}$ http://veritas.sao.arizona.edu/ 


\section{Introduction}

There are strong observational evidences that support the existence of dark matter (DM) in our universe, like the dynamics of galaxies and galaxy clusters, and the gravitational lensing effect.The abundance of this unknown component of the universe has been quantified to $84 \%$ of its total mass-density [1]. Assuming the hypothesis of DM being composed of weakly interacting massive particles (WIMPs), annihilation or decay of DM into Standard Model particles opens the possibility of indirectly detecting DM through the observation of these products, amongst them $\gamma$-ray photons (see [2]).The expected flux of prompt $\gamma$-ray emission from DM annihilation features a distinctive spectral shape primarily characterized by a cut-off at the WIMP mass.The preferred targets to search this DM spectral signature from are those regions showing the highest signal strength, by holding a large concentration of DM and/or being located close to the observer, like the Galactic Center and Halo, the dwarf spheroidal satellites of the Milky Way, and galaxy clusters. No clear DM signal has been detected so far in any of those objects [2].

High-resolution simulations of Milky Way-like DM halos indicate the presence of substructure down to the smallest resolved scales (see, e.g., [3]). Not all of these subhalos may have accumulated significant amounts of baryonic matter and would therefore be invisible to astronomical observations from radio to X-ray energies. Assuming a self-annihilating WIMP in the GeV-TeV mass range, these subhalos would be only visible at $\gamma$-ray energies. Since the emission from WIMP annihilation is expected to be steady, such hypothetical sources would be found in deep sky $\gamma$-ray surveys, and could already be part of the collection of Fermi-Large Area Telescope (LAT) sources showing no conventional counterpart at any other wavelengths [4]. If the distinct spectral cut-off at the WIMP mass is located at energies too high to be measurable by Fermi-LAT, imaging atmospheric Cherenkov telescopes (IACTs) like VERITAS may be crucial to identify such a DM signature. Several searches for DM subhalos in the HE $\gamma$-ray band have been conducted (see, e.g., [5; 6; 7]) and some of them have already triggered follow-up observations by IACTs [8; 9].

This contribution focuses on the observations of two DM subhalo candidates in the HE and VHE $\gamma$-ray bands. Section 2 describes the source selection, while the VERITAS VHE $\gamma$-ray and Fermi-LAT HE $\gamma$-ray observations and data analyses are contained in Sections 3 and 4 respectively. In Section 5 the results are discussed. A brief summary and outlook can be found in Section 6.

\section{Source selection}

The Second Fermi-LAT Catalog (2FGL) contains 1873 HE $\gamma$-ray sources detected by the LAT instrument after the first 24 months of observations, 576 sources lacking any clear association. We adapted the selection criteria from [8] to find good DM subhalo candidates among the latter unassociated sources. We requested our candidates: I. to be located at high Galactic latitudes $(|b|>$ $10^{\circ}$ ); II. not to present a variable flux; III. not to have potential counterparts at other wavelengths; IV. to be observable from VERITAS latitude with a maximum culmination zenith angle of $40^{\circ}$. To satisfy criterium III. we examined the $95 \%$ containment region of the candidates, looking for cataloged sources in the HEASARC Archive ${ }^{1}$ and analyzed Swift-XRT ${ }^{2}$ exposures when available. In addition, we estimated the required observation time for a $5 \sigma$ detection with VERITAS, based

\footnotetext{
1 http://heasarc.gsfc.nasa.gov/ 2 http://swift.gsfc.nasa.gov/
} 

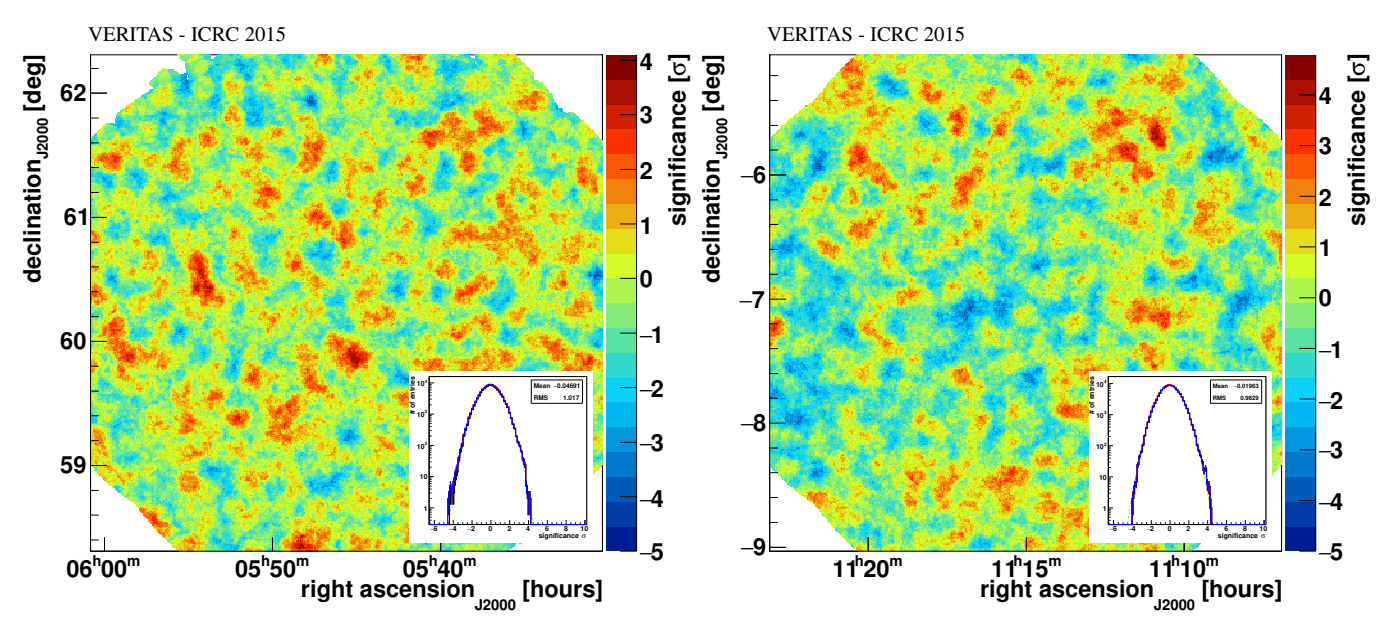

Figure 1: VERITAS significance sky maps of the regions centered on 2FGL J0545.6+6018 (left panel) and 2FGL J1115.0-0701 (right panel). Both significance distributions are well described by the hypothesis of background fluctuations, as illustrated by the significance histograms insets.

on a 2FGL Catalog flux extrapolation to the VHE range, discarding candidates that would require more than 50 hours of VERITAS time to be detected. We ended up with two best candidates, namely, 2FGL J0545.6+6018 and 2FGL J1115.0-0701. It is worth stressing that, by the time these sources were proposed for VERITAS observations, the 2FGL Catalog was the most updated publicly released catalog of Fermi-LAT point like sources. Thus, 2FGL Catalog denominations for our DM subhalo candidates will be used throughout the text.

\section{VERITAS observations and data analysis}

The Very Energetic Radiation Imaging Telescope Array System [10] is a ground-based $\gamma$-ray telescope array located at the Fred Lawrence Whipple Observatory in southern Arizona (31 40N, $11057 \mathrm{~W}, 1.3 \mathrm{~km}$ a.s.1.). The array consists of four imaging atmospheric-Cherenkov telescopes, each employing a tessellated $12 \mathrm{~m}$ Davies-Cotton reflector instrumented with a photomultipliertube camera with a $3.5^{\circ}$ field of view. VERITAS is designed to detect emission from astrophysical objects in the energy range from $85 \mathrm{GeV}$ to greater than $30 \mathrm{TeV}$, with a nominal sensitivity sufficient to detect, at the $5 \sigma$ level, a steady point-like source with $1 \%$ of the Crab Nebula flux in approximately $25 \mathrm{hrs}$. VERITAS has an energy resolution of $15 \%$ and an angular resolution (68\% containment) of $0.1^{\circ}$ at $1 \mathrm{TeV}$.

The VERITAS observations of 2FGL J0545.6+6018 and 2FGL J1115.0-0701 presented here were made under clear, moonless skies between October 2013 and March 2015, all of them after the 2012 upgrade of the VERITAS cameras and trigger system. After data-quality selection, 2FGL J0545.6+6018 observations amount to $8.5 \mathrm{hrs}$ performed at an average elevation of $61^{\circ}$, while $2 \mathrm{FGL}$ $\mathrm{J} 1115.0-0701$ observations span $13.8 \mathrm{hrs}$ at an average elevation of $51^{\circ}$. The data were acquired in the so called wobble (also known as false source) observation mode. The shower images from each telescope are calibrated, cleaned, and parametrized using a momentum analysis. These parameters are utilized to discriminate $\gamma$-ray like events against hadronic events applying multivariate analysis techniques, and are also combined to estimate the arrival direction and energy of the original $\gamma$-ray. The event selection cuts are optimized a priori over a Crab Nebula data set. 


\begin{tabular}{|c|c|c|c|c|c|c|c|}
\hline Source & $E_{t h}[\mathrm{GeV}]$ & $N_{\text {on }}$ & $N_{o f f}$ & $N_{e x c}$ & $\sigma$ & $N_{e x c}^{U L(95 \% \text { c.l. })}$ & $N_{e x c}^{U L(99 \% c . l .)}$ \\
\hline \multirow{3}{*}{ 2FGL J0545.6+6018 } & 150 & 327 & 5836 & $-16.2 \pm 18.6$ & -0.9 & 24.0 & 44.1 \\
\hline & 250 & 94 & 1704 & $-6.7 \pm 10.0$ & -0.7 & 28.4 & 52.0 \\
\hline & 450 & 24 & 536 & $-8.1 \pm 5.1$ & -1.5 & 3.3 & 8.4 \\
\hline \multirow{3}{*}{ 2FGL J1115. $\pm 0-0701$} & 150 & 673 & 10024 & $-4.7 \pm 26.8$ & -0.2 & 99.7 & 59.2 \\
\hline & 250 & 144 & 2112 & $0.5 \pm 12.4$ & 0.0 & 53.6 & 32.0 \\
\hline & 450 & 31 & 488 & $-2.6 \pm 5.8$ & -0.4 & 21.0 & 12.2 \\
\hline
\end{tabular}

Table 1: Upper limit to the number of excess events for three different energy thresholds $E_{t h}$. $N_{o n}$ and $N_{\text {off }}$ are the number of gamma-like events in the signal and background regions respectively, $N_{\text {exc }}$ being the number of excess event in the signal region. The significance $\sigma$ is computed applying Eq. 17 in [12].

\begin{tabular}{ccccccccc}
\hline & \multicolumn{9}{c}{ E } & \multicolumn{2}{c}{ Integral } & \multicolumn{2}{c}{ Differential } \\
& \multicolumn{3}{c}{$\mathrm{E}>150 \mathrm{GeV}$} & \multicolumn{2}{c}{$\mathrm{E}>250 \mathrm{GeV}$} & $\mathrm{E}>450 \mathrm{GeV}$ & $\mathrm{E}_{0}=1 \mathrm{TeV}$ \\
\hline & $\mathrm{c} .1$. & {$\left[\mathrm{cm}^{-2} \mathrm{~s}^{-1}\right]$} & {$[\mathrm{C} . \mathrm{U}]$.} & {$\left[\mathrm{cm}^{-2} \mathrm{~s}^{-1}\right]$} & {$[\mathrm{C} . \mathrm{U}]$.} & {$\left[\mathrm{cm}^{-2} \mathrm{~s}^{-1}\right]$} & {$[\mathrm{C} . \mathrm{U}]$.} & {$\left[\mathrm{TeV}^{-1} \mathrm{~cm}^{-2} \mathrm{~s}^{-1}\right]$} \\
\hline \multirow{2}{*}{ 2FGL J0545.6+6018 } & $95 \%$ & $1.95 \times 10^{-12}$ & $0.6 \%$ & $0.95 \times 10^{-12}$ & $0.6 \%$ & $0.16 \times 10^{-12}$ & $0.2 \%$ & $1.88 \times 10^{-13}$ \\
& $99 \%$ & $3.57 \times 10^{-12}$ & $1.0 \%$ & $1.69 \times 10^{-12}$ & $1.0 \%$ & $0.42 \times 10^{-12}$ & $0.6 \%$ & $2.38 \times 10^{-13}$ \\
\hline \multirow{2}{*}{ 2FGL J1115.0-0701 } & $95 \%$ & $2.15 \times 10^{-12}$ & $0.6 \%$ & $0.88 \times 10^{-12}$ & $0.5 \%$ & $0.31 \times 10^{-12}$ & $0.4 \%$ & $2.11 \times 10^{-13}$ \\
& $99 \%$ & $3.62 \times 10^{-12}$ & $1.0 \%$ & $1.48 \times 10^{-12}$ & $0.9 \%$ & $0.53 \times 10^{-12}$ & $0.8 \%$ & $3.54 \times 10^{-13}$ \\
\hline
\end{tabular}

Table 2: Upper limits to the integral fluxes for three different energy thresholds. The upper limits to the differential flux are computed for a pivot energy of $1 \mathrm{TeV}$.

We find no evidence for $\gamma$-ray emission above VERITAS energy threshold in any of the observed fields, as illustrated by the significance maps shown in Fig. 1. We note that the distribution of significances in either fields are compatible with pure background fluctuations. It is worth mentioning that the sources' coordinates in the 3FGL Catalog do not significantly change with respect to the values provided in the 2FGL Catalog (and used for VERITAS observations). The angular distance between both sets of coordinates are 3 arcmin and 4 arcsec for 2FGL J0545.6+6018 and 2FGL J1115.0-0701 respectively, well below VERITAS angular resolution.

The results from the VERITAS data analyses are summarized in Table 1, where observations significances and upper limits to the number of excess events coming from each source can be found, and Table 2, where integral and differential upper limits to the VHE $\gamma$-ray flux coming are listed. For the calculation of the upper limits to the number of excess events we applied the Rolke et al. method [11]. For the calculation of the upper limits to the flux we assumed power-law spectra with spectral indices of -2.0 and -2.1 for 2FGL J0545.6+6018 and 2FGL J1115.0-0701 respectively ${ }^{3}$. We constrain the integral flux of the sources in the VHE $\gamma$-ray domain to be below $1 \%$ of the Crab Nebula integral flux at a $95 \%$ confidence level.

\section{Fermi-LAT observations and data analysis}

The LAT is a space-based electron-positron pair-conversion instrument on board the Fermi $\gamma$-ray Space Telescope [13]. It is sensitive to $\gamma$ rays from $20 \mathrm{MeV}$ to more than $300 \mathrm{GeV}$. The LAT has a field of view of $2.4 \mathrm{sr}$ and operates primarily in an all-sky survey mode, covering the entire sky approximately every three hours.

We examined 6.7 years of LAT data (2008-07-31 to 2015-04-02, dubbed 7-years analysis henceforth) selecting reprocessed Pass 7 events in the energy range between $100 \mathrm{MeV}$ and $300 \mathrm{GeV}$.

\footnotetext{
3 These spectral indices are the best fit values to power law spectra from dedicated LAT analyses presented in Sec. 4.
} 


\begin{tabular}{|c|c|c|c|c|c|}
\hline & Analysis & $E_{0}$ & $N$ & $\alpha$ & $\beta$ \\
\hline & & {$[\mathrm{MeV}]$} & {$\left[\times 10^{-13} \mathrm{MeV}^{-1} \mathrm{~cm}^{-2} \mathrm{~s}^{-1}\right]$} & & \\
\hline \multirow{3}{*}{ 2FGL J0545.6+6018 } & 2FGL & 2292 & $(1.6 \pm 0.3) \times 10^{-13}$ & $1.95 \pm 0.11$ & $\mathrm{n} / \mathrm{a}$ \\
\hline & 3FGL & 1913 & $(4.0 \pm 0.5) \times 10^{-13}$ & $1.79 \pm 0.13$ & $0.30 \pm 0.09$ \\
\hline & 7-Years & 1913 & $(3.7 \pm 0.4) \times 10^{-13}$ & $1.76 \pm 0.12$ & $0.33 \pm 0.08$ \\
\hline \multirow{3}{*}{ 2FGL J1115.0-0701 } & 2FGL & 7491 & $(7.8 \pm 2.7) \times 10^{-15}$ & $1.60 \pm 0.28$ & $\mathrm{n} / \mathrm{a}$ \\
\hline & 3FGL & 1947 & $(1.7 \pm 0.2) \times 10^{-13}$ & $2.11 \pm 0.11$ & $\mathrm{n} / \mathrm{a}$ \\
\hline & 7-Years & 1947 & $(1.3 \pm 0.1) \times 10^{-13}$ & $2.13 \pm 0.10$ & $\mathrm{n} / \mathrm{a}$ \\
\hline
\end{tabular}

Table 3: Spectral parameters for 2FGL J0545.6+6018 and 2FGL J1115.0-0701 coming from the 2FGL and 3FGL Catalogs as well as our dedicated 7-year analysis. For sources showing a power-law best fit to their spectra these are parametrized as $d N / d E=N\left(E / E_{0}\right)^{-\alpha}$, while for sources best described by a log-parabola their spectra are parametrized as $d N / d E=N\left(E / E_{0}\right)^{-\alpha-\beta \log \left(E / E_{0}\right)}$. All errors are statistical.
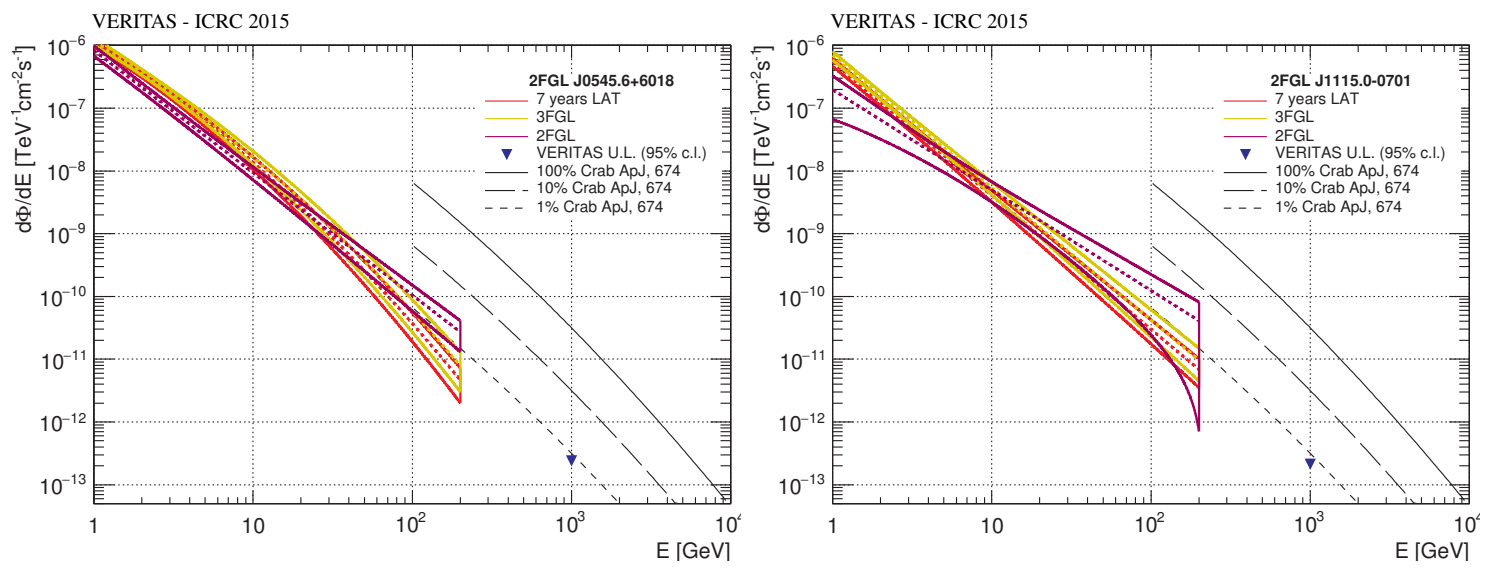

Figure 2: Fermi-LAT spectrum and $1 \sigma$ confidence contour of the fit, together with the VERITAS differential flux upper limits (95\% c.l.) for 2FGL J0545.6+6018 (left) and 2FGL J1115.0-0701 (right). The Crab Nebula differential spectrum in the VHE $\gamma$-ray band [14] at 100\%, 10\%, and $1 \%$ levels is shown as reference.

We selected class 2 events recorded at zenith angles below $100^{\circ}$ during good time intervals. We analyzed regions of interest of $10^{\circ}$ radius centered on the updated 3FGL coordinates of the sources. The data were analyzed using the Fermi Science Tools version v9r33p0-fssc-20140520.

Our 7-year analysis confirms the spectral characterization found in the 3FGL Catalog for the two sources of interest, and disfavors the spectral characterization found in the 2FGL Catalog. The collection of spectral parameters for both 2FGL J0545.6+6018 and 2FGL J1115.0-0701 are shown in Table 3. The agreement between our analyses and the 3FGL Catalog is also illustrated in Fig. 2.

In addition, we independently confirm the variable nature of the $\gamma$-ray emission from 2FGL J1115.0-0701 as first reported in the 3FGL Catalog. More specifically, the source presented a high activity state in mid January 2011 lasting for approximately two weeks with integral fluxes of $\sim 4 \times 10^{-9} \mathrm{~cm}^{-2} \mathrm{~s}^{-1}$ above $1 \mathrm{GeV}(T S=37)$, while its $T S$ value for the last 12 months of analyzed data is below 10 for energies larger than $100 \mathrm{MeV}$. The difference $(>1 \sigma)$ in the normalization factors between our analysis and the 3FGL Catalog nominal value could be attributed to the apparent fade off of 2FGL J1115.0-0701, while the spectral indices are compatible within errors.

\section{Results}

The flux variability for 2FGL J1115.0-0701 first noted in the 3FGL Catalog and later verified 
by our dedicated LAT analysis completely rejects the hypothesis of this source being a DM subhalo. Our 7-years analysis showed a high flux state with approximately two weeks duration compatible with an integral flux above $1 \mathrm{GeV}$ of $\sim 4 \times 10^{-9} \mathrm{~cm}^{-2} \mathrm{~s}^{-1}$ (14\% of the inverse Compton component of the Crab Nebula in the HE $\gamma$-ray band). VERITAS constraints to 2FGL J1115.0-0701 VHE flux would rule out the strict extrapolation of the 2FGL and 3FGL spectra into the VHE domain as described in Table 3. The extrapolation of the 7-years spectral characterization is not ruled out by the VERITAS constraints but it is in strong tension with them. In addition, VERITAS upper limits are still compatible within the extrapolated $1 \sigma$ confidence contour coming from each of those analyses. The tension between the VERITAS limits and our 7-years LAT analysis might favor some spectral softening at VHE which, if attributed to attenuation due to extragalactic background light absorption, together with the variable HE flux of the source and its high Galactic latitude, might support a distant blazar hypothesis as the nature of this source. However, it is also possible that the source was actually in a low state during part or all of the VERITAS observations, correlated to the lack of activity observed during the last 12 months of analyzed LAT data. In such a scenario nothing could be said in regards to the spectral softening of 2FGL J1115.0-0701 in the VHE band.

While VERITAS limits rule out the direct extrapolation to the VHE domain of the 2FGL Catalog spectral description of 2FGL J0545.6+6018, they do not constrain at all the log parabola description from the 3FGL Catalog and our 7-years analysis. Following up with the hypothesis of 2FGL J0545.6+6018 being a DM subhalo we interpret its SED in the $\gamma$-ray band to be purely originated by annihilation of WIMPs in the object. We model the differential flux as in Eq. 5.1, where $\left\langle\sigma_{a n n} v\right\rangle$ is the thermally-averaged self-annihilation cross-section, $m_{\text {WIMP }}$ is the WIMP mass, $d N_{\gamma}^{i} / d E$ is the photon yield per annihilation through channel $i, B^{i}$ is the branching ratio for channel $i$, and $\rho$ represents the density distribution of DM. We assume a value for the thermally-averaged self-annihilation cross-section of $\left\langle\sigma_{a n n} v\right\rangle=2.2 \times 10^{-26} \mathrm{~cm}^{3} \mathrm{~s}^{-1}[15]$ and consider the parametrizations from [16] as coded in the DAMASCO package ${ }^{4}$ for the photon yield per annihilation. Thus, our model contains two free parameters, $m_{\text {WIMP }}$ from the particle physics factor and the astrophysical factor $J$.

$$
\phi(E, \Delta \Omega)=\phi^{P P}(E) \times J(\Delta \Omega)=\underbrace{\frac{1}{4 \pi} \frac{\left\langle\sigma_{a n n} v\right\rangle}{2 m_{D M}^{2}} \sum_{i=1}^{n} B^{i} \frac{d N_{\gamma}^{i}}{d E} d E}_{\text {Particlephysicsfactor }} \times \underbrace{\int_{\Delta \Omega, \text { l.o.s. }} \rho^{2}(r(s, \Omega)) d s d \Omega}_{\text {Astrophysicalfactor }} .
$$

Four channels are considered: annihilation into $b \bar{b}, \mathrm{~W}^{+} W^{-}, \mu^{+} \mu^{-}$, and $\tau^{+} \tau^{-}$, with branching ratios $B^{i}=1$ in all scenarios. While the fit did not converge for the $\mu^{+} \mu^{-}$ channel and the goodness of fit for the $\tau^{+} \tau^{-}$ is really poor (the hypothesis of DM annihilating into $\tau$ particles is rejected at $\alpha<0.01$ ), the fits to annihilation into $b \bar{b}, W^{+} W^{-}$pro-

\begin{tabular}{lcccc}
\hline Channel & $\begin{array}{c}m_{\text {WIMP }} \\
{[\mathrm{GeV}]}\end{array}$ & $\begin{array}{c}\log _{10}(J) \\
{\left[\log _{10}\left(\mathrm{GeV}^{2} \mathrm{~cm}^{-5}\right)\right]}\end{array}$ & $\chi^{2} /$ d.o.f. & p-value \\
\hline$b \bar{b}$ & $78.3 \pm 11.6$ & $20.8_{-0.3}^{+0.2}$ & 1.02 & 0.36 \\
$W^{+} W^{-}$ & $89.2 \pm 13.7$ & $20.9_{-0.3}^{+0.2}$ & 0.74 & 0.48 \\
$\tau^{+} \tau^{-}$ & $18.0 \pm 0.3$ & $20.3_{-0.1}^{+0.1}$ & 14.69 & $4 \times 10^{-7}$ \\
\hline
\end{tabular}

Table 4: Summary of 2FGL J0545.6+6018 SED fit to $\mathrm{DM}$ annihilation spectra. We assume $\left\langle\sigma_{a n n} v\right\rangle=2.2 \times$ $10^{-26} \mathrm{~cm}^{3} \mathrm{~s}^{-1}$ for the estimation of the $J$-factors. vide with decently good fits. The best-fit values for the WIMP mass and the astrophysical factor together with their statistical errors can be found in Table 4 .

\footnotetext{
4 http://cta.gae.ucm.es/gae/damasco
} 
The most favored annihilation channel in terms of the goodness-of-fit is $W^{+} W^{-}$, with a $\chi_{\text {d.o.f }}^{2}=0.74$, although the fit result is in slight tension with the lowest-energy upper limit in the SED as showed in Fig. 3. On the other hand, annihilation into $b \bar{b}$, with a $\chi_{\text {d.o.f }}^{2}=1.04$, is compatible with the lowestenergy upper limit in the SED. We note that the later annihilation channel and its associated WIMP mass of $\sim 89 \mathrm{GeV}$ are similar to the results presented in [7] for a different set of DM subhalo candidates, and in reasonable agreement with the Fermi-LAT Galactic Center excess DM modeling in [17]. The estimated $J$-factors are one order of magnitude above the nominal $J$-factor for the most promising ultra-faint dwarf spheroidal

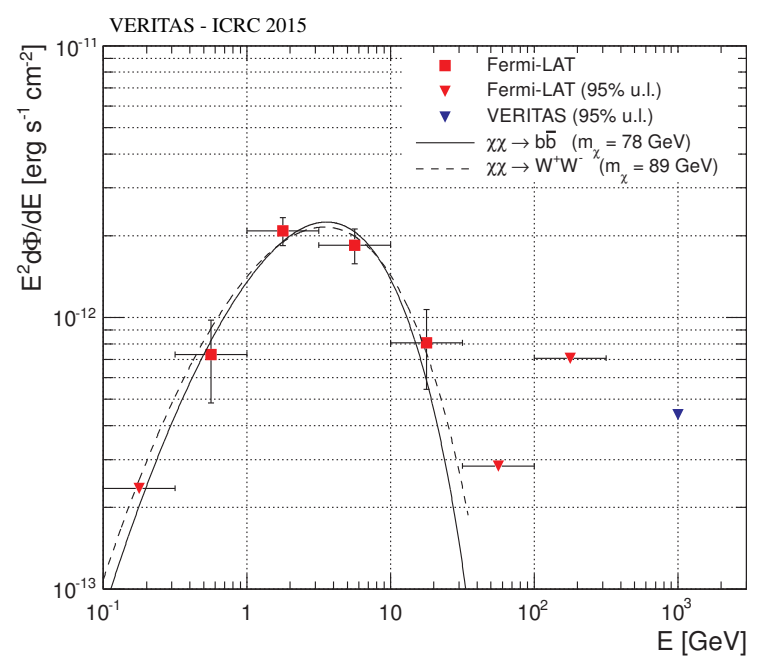

Figure 3: 2FGL J0545.6+6018 spectral energy distribution. The two best-fit annihilation spectra are shown. galaxy in [18], namely Ursa Mayor $\mathrm{II}^{5}$.The most constraining limits in this energy range come from the analysis of Fermi-LAT data on dwarf spheroidal galaxies [19]. For annihilation into $b \bar{b}$ and $m_{W I M P}=78 \mathrm{GeV}$, they found $\left\langle\sigma_{\text {ann }} v\right\rangle<$ $1.0 \times 10^{-25} \mathrm{~cm}^{3} \mathrm{~s}^{-1}$ at $95 \% \mathrm{cl}$. Similarly, for annihilation into $W^{+} W^{-}$and $m_{W I M P}=89 \mathrm{GeV}$, they found $\left\langle\sigma_{a n n} v\right\rangle<1.6 \times 10^{-25} \mathrm{~cm}^{3} \mathrm{~s}^{-1}$ at $95 \% \mathrm{cl}$. Therefore, although in tension with the latter limits, our results are not ruled out by them.

\section{Summary and outlook}

We have presented a search for DM subhalo candidates in the 2FGL Catalog and the results from the observations with VERITAS of two promising candidates, 2FGL J0545.6+6018 and 2FGL J1115.0-0701, along with a dedicated 7-years LAT data for both sources.

2FGL J1115.0-0701 flux variability and high Galactic latitude opens the possibility of this source being a blazar. We encourage the monitoring of 2FGL J1115.0-0701 HE $\gamma$-ray activity searching for potential flaring events which could drive its VHE $\gamma$-ray component, depending on the source's redshift, into the sensitivity reach of the current generation of IACTs.

We interpreted 2FGL J0545.6+6018 SED in terms of DM annihilating via different channels. Annihilation into $b \bar{b}$ and $W^{+} W^{-}$fit the source's $\gamma$-ray emission reasonably well, providing WIMP masses of $78.3 \mathrm{GeV}$, and $89.2 \mathrm{GeV}$ correspondingly. While 2FGL J0545.6+6018 $\gamma$-ray emission has been interpreted in terms of a DM signal, less exotic explanations compatible with its spectral shape can be offered, like attributing its emission to a radio-faint $\gamma$-ray pulsar. Multiwavelength follow-up observations are encouraged in order to elucidate the real nature of this enigmatic source.

It is worth mentioning the impact that the next-generation of IACTs, represented by the Cherenkov Telescope Array (CTA, [20]), may have on this kind of searches. CTA is expected

\footnotetext{
$\left.\overline{\log _{10}\left(J_{U M a} I I\right.}\right)=19.9_{-0.5}^{+0.7}$ for an integration angle of $0.5^{\circ}$ (the $J$-factor units are $\mathrm{GeV}^{2} \mathrm{~cm}^{-5}$ ).
} 
to improve current-generation IACTs' sensitivity by a factor of ten which, in conjunction with its survey capabilities, will increase the likelihood to serendipitously discover new VHE $\gamma$-ray sources and, potentially, DM subhalos.

\section{Acknowledgments}

This research with VERITAS is supported by grants from the U.S. Department of Energy Office of Science, the U.S. National Science Foundation and the Smithsonian Institution, and by NSERC in Canada. We acknowledge the excellent work of the technical support staff at the Fred Lawrence Whipple Observatory and at the collaborating institutions in the construction and operation of the instrument. DN acknowledges partial support from the National Aeronautics and Space Administration through the Fermi Guest Investigator Program. The VERITAS Collaboration is grateful to Trevor Weekes for his seminal contributions and leadership in the field of VHE gamma-ray astrophysics, which made this study possible.

\section{References}

[1] Planck Collaboration, P. A. R. Ade, et al., Planck 2015 results. XIII. Cosmological parameters, ArXiv e-prints (Feb., 2015) [arXiv:1502.0158].

[2] J. Conrad, J. Cohen-Tanugi, and L. E. Strigari, WIMP searches with gamma rays in the Fermi era: challenges, methods and results, ArXiv e-prints (Mar., 2015) [arXiv: 1503.0634].

[3] J. Diemand, M. Kuhlen, et al., Clumps and streams in the local dark matter distribution, Nature 454 (2008) 735-738, [arXiv:0805.1244].

[4] L. Pieri, J. Lavalle, G. Bertone, and E. Branchini, Implications of high-resolution simulations on indirect dark matter searches, 83 (Jan., 2011) 023518, [arXiv: 0908 .0195].

[5] D. Nieto, V. Martínez, et al., A search for possible dark matter subhalos as IACT targets in the First Fermi-LAT Source Catalog, $3^{\text {rd }}$ Fermi Symposium, Rome (Oct., 2011) [arXiv: 1110 . 4744].

[6] H.-S. Zechlin and D. Horns, Unidentified sources in the Fermi-LAT second source catalog: the case for DM subhalos, JCAP 11 (Nov., 2012) 50, [arXiv:1210.3852].

[7] B. Bertoni, D. Hooper, and T. Linden, Examining The Fermi-LAT Third Source Catalog In Search Of Dark Matter Subhalos, ArXiv e-prints (Apr., 2015) [arXiv: 1504 .0208].

[8] D. Nieto et al., The search for galactic dark matter clump candidates with Fermi and MAGIC, $32^{\text {nd }}$ International Cosmic Ray Conference, Beijing (Aug., 2011) [arXiv:1109.5935].

[9] A. Geringer-Sameth and for the VERITAS Collaboration, The VERITAS Dark Matter Program, $4^{\text {th }}$ Fermi Symposium, Monterrey, CA (2012) [arXiv:1303.1406].

[10] J. Holder et al., The first VERITAS telescope, Astroparticle Physics 25 (July, 2006) 391-401, [ast ro-ph / 0604119 ].

[11] W. A. Rolke, A. M. Lopez, and J. Conrad, Limits and confidence intervals in the presence of nuisance parameters, A551 (2005) 493-503, [physics/0403059].

[12] T.-P. Li and Y.-Q. Ma, Analysis methods for results in gamma-ray astronomy, ApJ 272 (1983) 317-324.

[13] Fermi-LAT Collaboration, W. Atwood et al., The Large Area Telescope on the Fermi Gamma-ray Space Telescope Mission, ApJ 697 (2009) 1071-1102, [arXiv: 0902.1089].

[14] MAGIC Collaboration, J. Albert et al., VHE Gamma-Ray Observation of the Crab Nebula and Pulsar with MAGIC, ApJ 674 (2008) 1037-1055, [astro-ph/0705.3244].

[15] G. Steigman, B. Dasgupta, and J. F. Beacom, Precise relic WIMP abundance and its impact on searches for dark matter annihilation, 86 (July, 2012) 023506, [arXiv:1204.3622].

[16] J. A. R. Cembranos, A. de La Cruz-Dombriz, et al., Photon spectra from WIMP annihilation, Phys.Rev.D 83 (Apr., 2011) 083507-+, [hep-ph/1009.4936].

[17] F. Calore, I. Cholis, C. McCabe, and C. Weniger, A tale of tails: Dark matter interpretations of the Fermi GeV excess in light of background model systematics, 91 (Mar., 2015) 063003, [arXiv: 1411.4647].

[18] V. Bonnivard, C. Combet, et al., Dark matter annihilation and decay in dwarf spheroidal galaxies: The classical and ultrafaint dSphs, arXiv:1504.0204.

[19] Fermi-LAT Collaboration, Searching for Dark Matter Annihilation from Milky Way Dwarf Spheroidal Galaxies with Six Years of Fermi-LAT Data, ArXiv e-prints (Mar., 2015) [arXiv: 1503.0264 ].

[20] B. Acharya et al., Introducing the cta concept, Astroparticle Physics 43 (2013), no. 03 - 18. Seeing the High-Energy Universe with the Cherenkov Telescope Array - The Science Explored with the CTA. 